\title{
Avaliação de pavimento asfáltico utilizando a biblioteca RGPR da linguagem $\mathbf{R}$
}

Nicchetti,Tatiane A., UFBA; Schibelscky,Mariana L. N. O. S., UFBA; Botelho, Marco A. B., UFBA.

Copyright 2018, SBGf - Sociedade Brasileira de Geofísica

Este texto foi preparado para a apresentação no VIII Simpósio Brasileiro de Geofísica, Salinópolis, 18 a 20 de setembro de 2018. Seu conteúdo foi revisado pelo Comitê Técnico do VIII SimBGf, mas não necessariamente representa a opinião da SBGf ou de seus associados. É proibida a reprodução total ou parcial deste material para propósitos comerciais sem prévia autorização da SBGf.

\begin{abstract}
The objective of this paper is to show how the GPR can be used to asphaltic evaluation and how to process the data using a new library of language $R$ called RGPR. Google earth was used to asset the traveled distance as well as to guess the position of viaducts on the radargrama. Furthermore, sections of interest were chosen based on local anomalies. Along this paper, a sequence of processing tools were used, including time 0 correction, time gain and spacial filters. At the end, it's concluded that the use of this library could be a free substitute to other paid softwares.
\end{abstract}

\section{Introdução}

O Georadar é um método geofísico de superfície que utiliza ondas eletromagnéticas de alta frequência comumente descritas como pertencentes a faixa de 10$1000 \mathrm{MHz}$ - que mapeia estruturas rasas em subsolo ou localiza objetos enterrados tais como adutoras.

O funcionamento do GPR consiste em irradiar ondas eletromagnéticas ao solo cuja transmissão depende das propriedades do meio, notadamente a condutividade elétrica e a permissividade dielétrica. Essas propriedades sofrem grandes modificações com o teor de água presente no solo.

A teoria do Georadar parte do princípio que o sinal recebido é o mesmo que o emitido pela antena, o que contraria a realidade por desprezar o efeito da perda de energia com o tempo de propagação. Esse fenômeno é decorrente principalmente devido à absorção (perda de amplitude do pulso como resultado da conversão da energia eletromagnética em calor, também chamada de efeito joule), à dispersão (alargamento do pulso a medida que ele se propaga) e ao espalhamento geométrico (diminuição da densidade de energia relacionada a propagação da frente de onda, a qual depende da distribuição de velocidades do meio).

Atualmente o GPR é conhecido no mercado como um meio de mapeamento não-destrutivo de inúmeras aplicações, como detecção de fraturas em rochas, investigações (estratigráfica, hidrogeológica e arqueológica), cem como outras aplicações na área de engenharia geotécnica.
A avaliação do pavimento asfáltico é imprescindível, já que as rodovias são o principal meio de transporte no Brasil. Uma pavimentação do tipo flexível (comumente associada a pavimentos asfálticos), é normalmente dividida em 4 componentes: base, sub-base, subleito e revestimento (Bernucci, 2008).

Com a evolução da tecnologia do georadar, a quantidade de softwares disponíveis no mercado aumentou de forma significativa. Infelizmente, pelo fato de muitos deles ainda serem pagos, o acesso a essas plataformas é dado de forma limitada para estudantes da graduação e outros profissionais interessados.

Com o intuito de facilitar o acesso e permitir uma maior dinamização do processamento GPR, é de extrema importância o surgimento de plataformas gratuitas, como o caso do RGPR.

\section{Aquisição}

O dado foi adquirido na Avenida Orlando Gomes localizado no bairro de Piatã, Salvador, Ba.

A antena utilizada foi o modelo 50104A de $1 \mathrm{GHz}$ da GSSI acoplada a um veículo. O percurso foi de aproximadamente $3.3 \mathrm{~km}$ no sentido orla-metrô do bairro da paz(percurso OG3). O trajeto do levantamento da seção passa sobre 3 (três) viadutos, como é possível observar na fig.1:

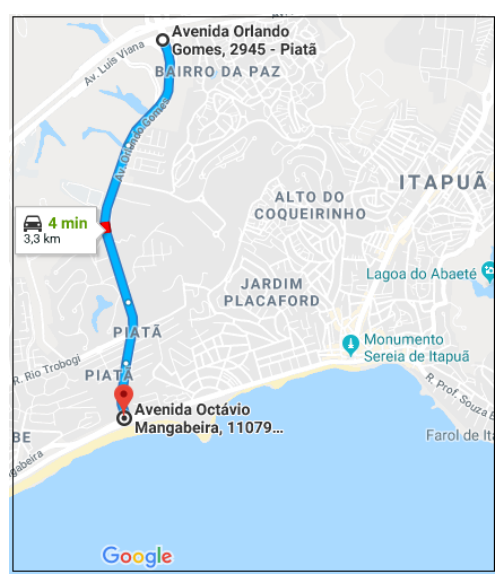

Fig. 1: Google maps mostrando o percurso. Coordenadas aproximadas: -12.953014,-38.385039 a -12.926873,-381671 
O dado apresenta 6405 traços, tendo $2 \mathrm{scan} / \mathrm{m}$. Para fazer a medição correta da distancia percorrida, um odômetro foi acoplado a roda do veiculo.

A estimativa da posição dos viadutos V-I, V-II e V-III também são dadas pelo Google Maps segundo a fig.2:

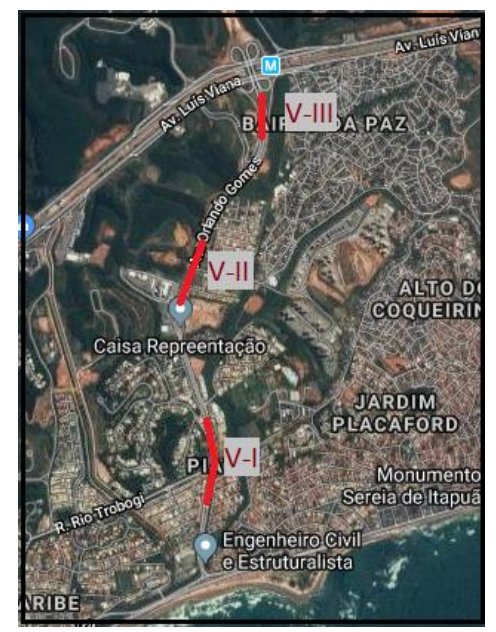

Fig. 2: Imagem do Google Maps mostrando a posição estimada dos viadutos

A posição relativa desses viadutos é dada utilizando o REFLEX2DQUICK (linhas em vermelho), enquanto os pontos de interesse foram encontrados e denominados de $A$ a $E$ (círculos verdes). A fig. 3 mostra a posição dessas seções de interesse em relação aos viadutos presentes na rodovia.

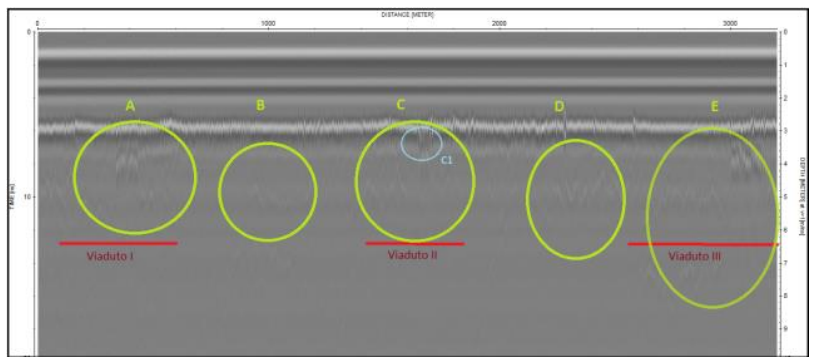

Fig. 3: Mostrando pontos de interesse e suas posições relativas aos viadutos.

\section{Processamento usando o RGPR}

O ponto está na posição compreendida entre 800 e $1200 \mathrm{~m}$ (traços de 1600 a 2400) no percurso OG3. Por não estar sobre nenhum viaduto, ele é ideal para analisar as feições do pavimento asfáltico. A velocidade estimada para esse material é de $0.13 \mathrm{~m} / \mathrm{ns}$ :

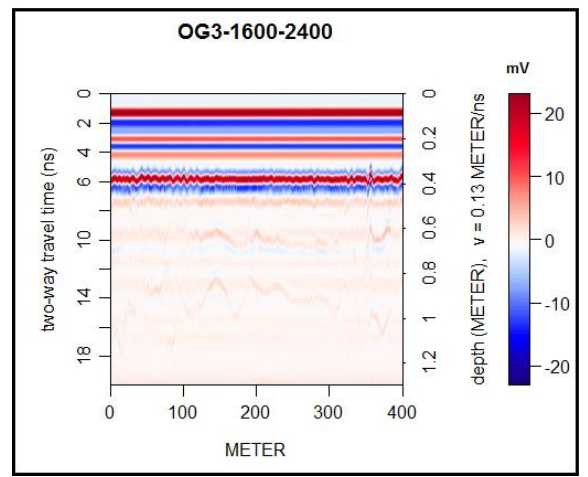

Fig. 4: visualização do ponto $B$ usando a biblioteca RGPR

Nem sempre o tempo 0 do radargrama corresponde ao tempo inicial. Como a antena estava suspensa, é preciso visualizar o arquivo de calibração da antena, para notar qual o tempo que corresponde a interface entre 0 ar e 0 pavimento. Nesse caso, o tempo $0=4.4 \mathrm{~ns}$, como pode ser observado pela Fig. 5:

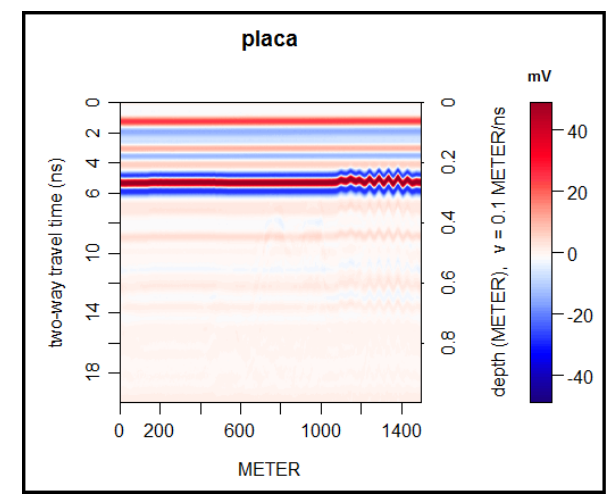

Fig. 5: arquivo de calibração da placa

O próximo passo é analisar um traço individualmente para notar se ele tem algum deslocamento devido ao acoplamento entre a antena transmissora e a receptora (DC shift) e, posteriormente, aplicar o Dewow para corrigir os efeitos devido a energia de baixa frequência. Como o traço não estava deslocado e o dewow não mostrou efeitos perceptíveis, essas etapas não serão detalhadas no processo desse trabalho.

Posteriormente, é possível analisar o espectro de frequência pela decomposição de Fourier, como indicado na fig.6: 


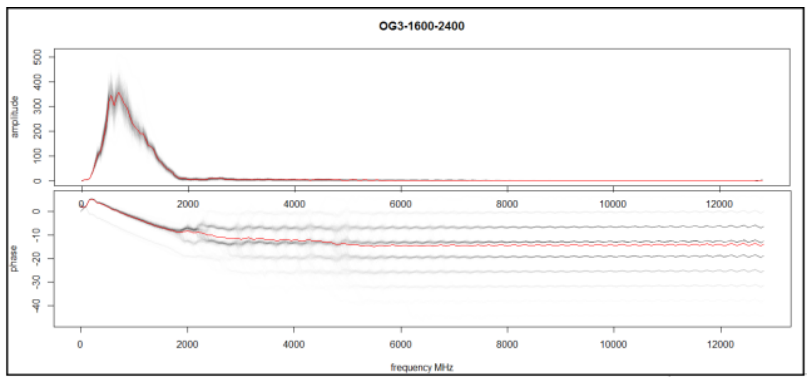

Fig. 6: A decomposição de Fourier na forma de gráfico de amplitude-frequência(acima) e de fase-frequência (abaixo)

Note que a concentração da distribuição da frequência esta próxima a frequência central da antena, que equivale a $1 \mathrm{GHz}$.

É possível notar que a partir de $1800 \mathrm{GHz}$, a frequência é relativamente constante, mostrando uma região com ruídos que devem ser atenuados utilizando um filtro passa alta.

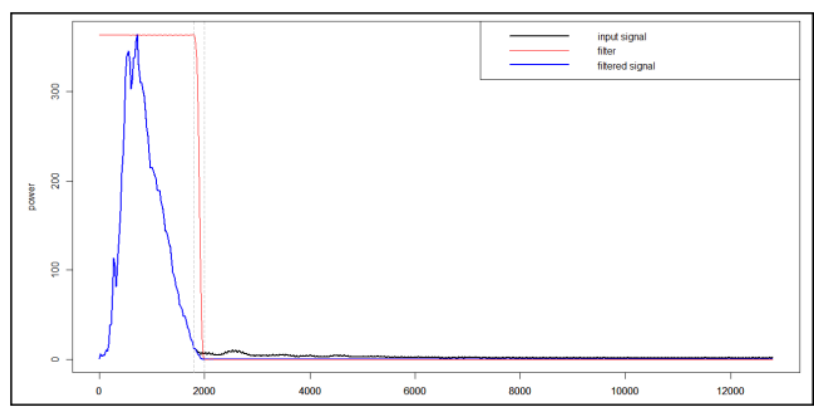

Fig. 7: Gráfico esquemático mostrando a amplitude do dado antes (em preto) e depois do filtro (em azul). Perceba que as informações provenientes após $2000 \mathrm{GHz}$ foram cortadas.

Para aplicar o ganho é interessante primeiro visualizar o logarítmico da amplitude pelo tempo como indicado pela fig. 8. Desse modo, é possível fazer uma estimativa preliminar de possíveis interfaces e decidir qual o melhor tipo de ganho a ser aplicado. Nesse caso, foi escolhido o ganho exponencial (power gain). O power gain deve ser constante ate $1 \mathrm{~ns}$, devendo ser aplicado até a faixa de 12 ns, quando começa o ruído.

Utiliza-se um "filtro da mediana" com o objetivo de percorrer o sinal, substituindo cada traço com a mediana dos traços vizinhos. É uma forma de reduzir ruídos pontuais.

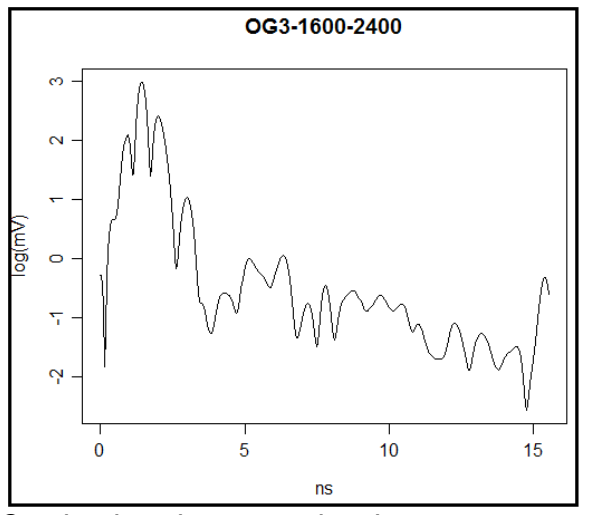

Fig. 8: O primeiro pico por volta de 1-2ns corresponde a interface entre $o$ ar e o pavimento. $O$ segundo pico por volta de 5 ns e o terceiro por volta de 10ns correspondem a feições que eu desejo destacar.

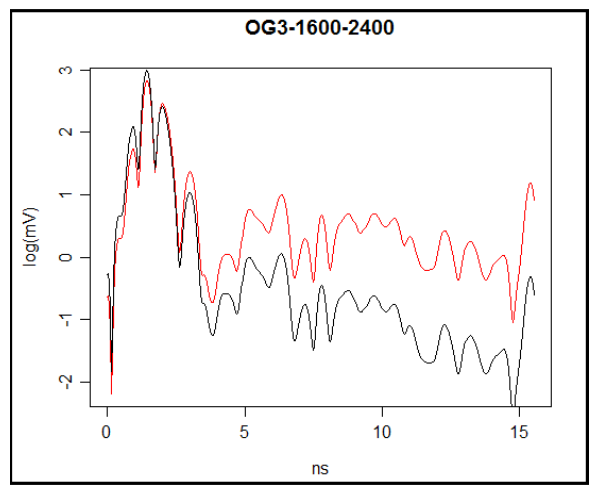

Fig. 9: Em vermelho o dado após o ganho e em preto o dado antes do ganho

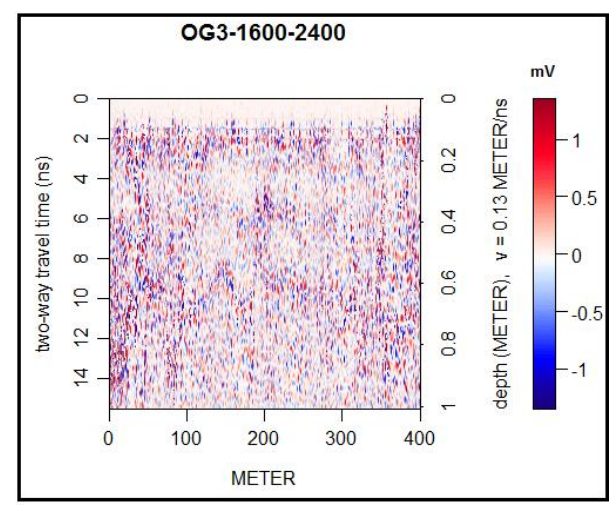

Fig. 10: O ruído eliminado durante o procedimento de horizontal averaging. 


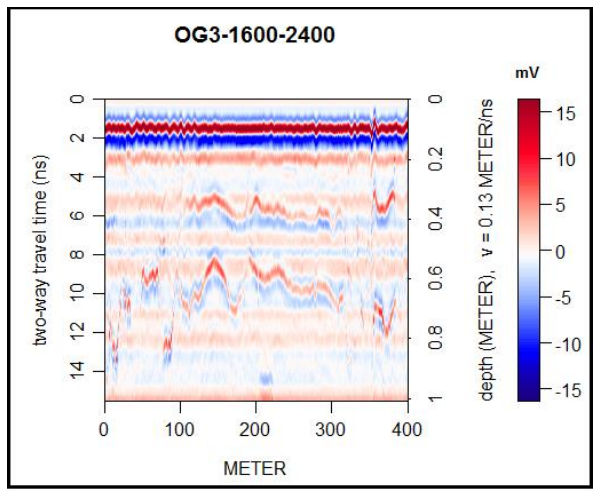

Fig. 11: visualização do dado após aplicação de todos os procedimentos anteriores.

A técnica de "horizontal averaging" (Fig.10) leva em consideração que as interfaces são contínuas e que os ruídos são aleatórios. Por esse motivo, eventos nãolineares são extraídos do radargrama, causando um maior suavização ("smoothing") do dado quanto maior for a janela de atuação (w). Essa técnica será utilizada de forma reversa, para extrair do dado as feições de interesse e permitir uma maior visualização. Por esse motivo, uma janela relativamente grande de $\mathrm{w}=100$ foi escolhida:

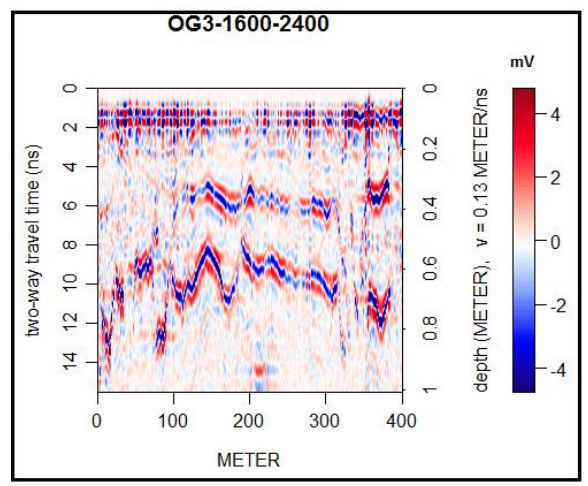

Fig. 12: Resultado final do processamento

\section{Resultados e conclusões}

É possível observar a presença de duas interfaces. A primeira corresponde a interface entre a base e subbase, enquanto a segunda entre a sub-base e o sub-leito, como mostra a fig. 13:

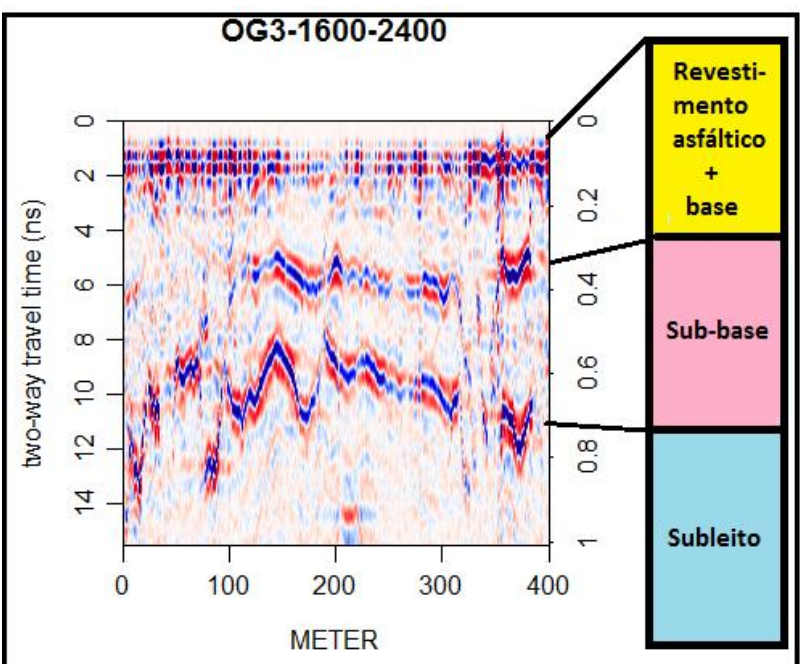

Fig. 13: Esquema da interpretação da seção. $O$ revestimento asfáltico + base teriam a espessura aproximada de $4 \mathrm{~cm}$. Uma espessura semelhante pode ser inferida para a camada de sub-base.

É importante salientar que a antena de 1 Ghz não teve resolução suficiente para determinar a espessura da camada asfáltica ou delinear a interface entre 0 revestimento asfáltico e a camada de base.

Quanto ao uso da biblioteca RGPR, é possível concluir que é uma alternativa viável e ao nível dos softwares convencionais, possibilitando que o operador tenha maior controle sobre o processo e possa visualiza-lo etapa por etapa. Como ainda esta em desenvolvimento, novas funções podem ser adicionados, incrementando sua utilidade.

\section{Agradecimentos}

Agradeço a professora Mariana e ao professor Botelho pela orientação deste trabalho.

Agradecimento especial ao professor Luis Edmundo e a toda equipe de geotécnica da UFBA, bem como a Manuel Huber por ter permitido a utilização da biblioteca RGPR.

Todas as imagens de satélite foram providas utilizando o Google Maps.

\section{Referências}

Annan, A. (1999) Practical processing of gpr data, Sensors and software, Ontario.

Bernucci, L. B. et al. Pavimentação Asfáltica: Formação Básica para Engenheiros, 1a Edição PETROBRAS. ABEDA, Rio de Janeiro, RJ, Brasil, 2008.

Cassidy, N.J. (2009) Ground Penetrating Radar Data Processing, Modelling and Analysis, Elsevier. University of wisconsin-Eau Claire, USA. In: Jol, Harry M. (2009) 
Ground Penetrating Radar: Theory and Applications, Elsevier. University of wisconsin-Eau Claire, USA.

Jol, Harry M. (2009) Ground Penetrating Radar: Theory and Applications, Elsevier. University of wisconsin-Eau Claire, USA.

SHRESTHA, Shanker Man et al. Signal processing of ground penetrating radar using super resolution technique. In: Radar Conference, 2001. Proceedings of the 2001 IEEE. IEEE, 2001. p. 300-305.

XAVIER, NETO. P. Processamento e interpretação de dados 2D e 3D de GPR: Aplicações no imageamento de feições cársticas e estruturas de dissolução no campo de petróleo de Fazenda Belém-CE. Programa de Pesquisa e Pós-Graduação em Geodinâmica e Geofísica, Universidade Federal do Rio Grande do Norte, Natal, Tese de Doutorado, v. 176, 2006. 